\title{
Causal impact of malnutrition on mortality among adults hospitalized for medical illness in sub-Saharan Africa: what is the role of severe sepsis?
}

\author{
Stephen B. Asiimwe ${ }^{1,3^{*}}$, Abdallah Amir ${ }^{1,2}$, Eric Vittinghoff ${ }^{3}$ and Conrad K. Muzoora ${ }^{2}$
}

\begin{abstract}
Background: In sub-Saharan Africa, malnutrition is associated with mortality in adults hospitalized for medical illness. However, it remains unclear whether this association is causal, and if causal, what the potential mediators are. We assessed whether malnutrition is causally related to mortality among hospitalized adults, and whether severe sepsis plays a mediating role.

Methods: We analyzed data from a cohort study of adults hospitalized for any medical illness in Uganda. We measured malnutrition using mid-upper arm circumference (MUAC). We used a directed acyclic graph to identify a minimum sufficient adjustment set of confounders in order to estimate the overall effects of malnutrition on mortality. We then used recently developed statistical methods to determine whether mortality in malnourished patients is mediated by severe sepsis.

Results: We analyzed data of 318 adults. Median age was 37 (interquartile range [IQR] 27 to 56 ), and $25 \%$ ( $n=80$ ) were malnourished according to MUAC. Malnourished patients were more likely to be HIV positive (64 \% versus $39 \%, p<0.001$ ), more severely ill (median MEWS 5, IQR 3 to 7 versus 4, IQR 2 to $6, P=0.003$ ), and to have both any sepsis (66 \% versus $39 \%, p<0.001)$ and severe sepsis (51\% versus $20 \%, P<0.001)$ compared to normally nourished patients. After adjusting for the modified early warning score at admission, tuberculosis, HIV status, education status, age, and sex, malnourished patients remained at 3.0-fold (95\% Cl: 1.5, 6.1, $P=0.002$ ) increased odds of having severe sepsis at admission, and at 2.1 -fold (95\% confidence interval [Cl]: 1.2, 3.7, $P=0.008$ ) increased odds of dying by 30 days post-admission. Only a small proportion of the effects of malnutrition on mortality were mediated by severe sepsis; overall, malnutrition increased the risk of death by 16.8 percentage points (95\% Cl: 4.1, 29.4), but only 1.1 percentage points $(95 \% \mathrm{Cl}:-2.1,4.4)$ absolute risk difference was mediated by severe sepsis.

Conclusion: Our data suggest that malnutrition increases mortality in adults hospitalized for medical illness; thus, interventions against malnutrition in this population may reduce mortality. As severe sepsis did not mediate a large proportion of the effects of malnutrition on mortality, future studies are recommended to investigate other potential mediators.
\end{abstract}

Keywords: Malnutrition, Hospitalized adults, Mortality, Severe sepsis, Causal impact, Mediation

\footnotetext{
* Correspondence: asiimwesteve@gmail.com

'Department of Medicine, Mbarara Regional Referral Hospital, P.O Box, 40, Mbarara, Uganda

${ }^{3}$ Department of Epidemiology and Biostatistics, University of California, San

Francisco, CA, USA

Full list of author information is available at the end of the article
} 


\section{Background}

In sub-Saharan Africa (SSA), mortality among adults hospitalized with medical illness is alarmingly high and deserves further investigation $[1,2]$. In a recent study in Zambia, in-hospital mortality was about $60 \%$ in patients admitted with severe sepsis [3]. There is urgent need for data identifying important risk factors for mortality and the mechanisms through which they cause death. Malnutrition and severe sepsis, both of which are common in the region, are two such risk factors $[4,5]$. We, as well as other investigators, have previously shown that malnutrition at admission was associated with mortality in adults hospitalized for medical illnesses $[4,6,7]$. However, it remains unclear whether this association is causal.

Although not yet in common use in the region, emerging epidemiologic techniques, such as directed acyclic graph (DAG)-based causal analysis [8], have potential to improve our understanding of risk factors for mortality in this setting. DAG-based techniques are particularly appealing in sub-Saharan Africa, as mortality risk factors in individual patients rarely occur in isolation [9]. Such studies can also guide selection of interventions by determining mediators of observed mortality risk and comparing likely benefits from alternative intervention approaches [10].

The association between malnutrition and mortality in this setting could be due to confounding, especially by HIV infection and/or tuberculosis (TB), but possibly also by other chronic illnesses. However, there is reason to suspect a causal effect of malnutrition on mortality. Since malnutrition increases susceptibility to infection [11-14], malnourished patients may be at higher risk of infectious complications such as severe sepsis, which can lead to death [15]. Malnutrition could also increase mortality through hypoglycemia, hypothermia, and anemia [15-17]. Malnourished patients getting any severe illness may also do worse than those who are not malnourished [18].

We used a DAG to identify a minimum sufficient adjustment set of confounders in order to estimate the overall effect of malnutrition on mortality among adults hospitalized for any medical illness. We then used recently developed statistical methods to estimate how much of the overall effect of malnutrition on mortality is mediated by severe sepsis.

\section{Methods}

\section{Setting and study population}

We analyzed data from an observational cohort study of hospitalized adults. The study occurred on the general medicine ward of Mbarara Regional Referral Hospital in south-Western Uganda. The setting and study population have been described elsewhere [6]. All patients being admitted for any medical illness during the study period (April to June 2011) were eligible for the study, which was set up to perform multiple nutritional assessments on each patient. However, those who were less than 18 (considered unable to provide informed consent) $(N=34)$ and those who declined to participate in the study $(N=3)$ were excluded. As we were able to perform the planned nutritional assessments on all consenting patients, no patient was excluded on this basis.

\section{Ethical approval}

All participants provided written informed consent. The study was approved by the Institutional Review Committee of Mbarara University of Science and Technology.

\section{Measurement of malnutrition}

For this report, we measured malnutrition at admission using the mid-upper arm circumference (MUAC). MUAC has been validated in adults for diagnosing malnutrition and shown to be highly correlated with the body mass index while predicting outcomes better [19-21]. MUAC is in general highly specific (up to $96 \%$ ), but can be poorly sensitive depending on the chosen cut-offs [22]. We used a non-stretchable MUAC tape to obtain MUAC as the circumference of either of the patient's arm at the midpoint between the olecranon and the acromion, with the patient seated or lying supine, and the rested arm flexed at $90^{\circ}$ at the elbow. We defined malnutrition as a MUAC $<19 \mathrm{~cm}$ for females and $<20 \mathrm{~cm}$ for males [19].

\section{Other predictor measurements}

We defined sepsis as the presence of suspected infection plus 2 or more of the systemic inflammatory response syndrome (SIRS) criteria (pulse $\geq 90$ beats/min; respiratory rate $\geq 20$ cycles /min; a temperature $\geq 38{ }^{\circ} \mathrm{C}$ or $\leq 36{ }^{\circ} \mathrm{C}$; and white blood cell count $\geq 12,000$ cells/cc or $<4,000$ cells/cc); severe sepsis was defined as the presence of sepsis plus at least 1 organ dysfunction (Glasgow coma score $[\mathrm{GCS}]<15$, systolic blood pressure $<90 \mathrm{mmHg}$, mean arterial pressure $<70 \mathrm{mmhg}$, or platelet counts $<100,000$ cells/cc) [23]. All temperature measurements were axillary. Diagnoses for infections and focus of infection were based on clinical suspicion and physical examination. We measured at admission, a random blood glucose (RBS) using a hand-held glucometer (Roche Diagnostics, Basel, Switzerland), and defined hypoglycemia as RBS $<4.5 \mathrm{mmol} / \mathrm{l}$ [24]. We also obtained a complete blood count (Beckman Coulter, Villepinte, France) defining (severe) anemia as a hemoglobin concentration $<8 \mathrm{~g} / \mathrm{dl}$ in both males and females [25]. HIV status was determined using a standard 3-test rapid testing algorithm (Determine, Abbott Laboratories, Abbott Park, IL; Statpak, Chembio Diagnostics, Medford, New York; and Unigold, Trinity 
Biotech, Bray, Ireland), and tuberculosis was defined as sputum smear positive pulmonary tuberculosis, or microscopically confirmed extra-pulmonary tuberculosis, or sputum smear negative tuberculosis as diagnosed through a consensus of at least two physicians [26]. All laboratory tests were performed at the Mbarara University Clinical Research Laboratory which participates in external quality assurance by the National Health Laboratory Service (Johannesburg, South Africa).

\section{Severity of underlying illness}

We measured severity of underlying illness using the Modified Early Warning Score (MEWS) which incorporates blood pressure, pulse, temperature, respiratory rate, and the neurological status at admission and strongly predicts mortality in hospitalized adults [27]. MEWS gives: 3 points for each of systolic blood pressure $<70$ mmhg, pulse rate $\geq 130$ beats $/ \mathrm{min}$, respiratory rate $\geq 30$ cycles/min, and GCS score $\leq 8 ; 2$ points for each of SBP $70-80$ or $\geq 200$, pulse $111-129$, respiratory rate $<9$ or $21-$ 29 , temperature $<35$ or $\geq 38.5$ Celsius, and GCS $9-13$; and a point each for SBP $80-100$, pulse $40-50$ or $101-110$, respiratory rate 16-20, and GCS score of 14 . The accrued total score can be incorporated into analyses, usually as a categorical variable [28].

\section{Outcome}

The outcome was 30-day mortality. All patients were followed in hospital to death or discharge, and after discharge, they were further followed using mobile telephone calls and out-patient clinic appointments to determine 30-day vital status.

\section{Data analysis}

We tested the association between malnutrition and patient characteristics at admission using chi-squared or Mann-Whitney-U tests as appropriate.

For the causal analysis, we identified confounders of the relationships between malnutrition, severe sepsis, and 30-day mortality using a directed acyclic graph (DAG) [8] (Fig. 1). In order to build the final DAG and the final adjusted model, we started by drawing a large conceptual framework including all variables that we considered to be associated with malnutrition, severe

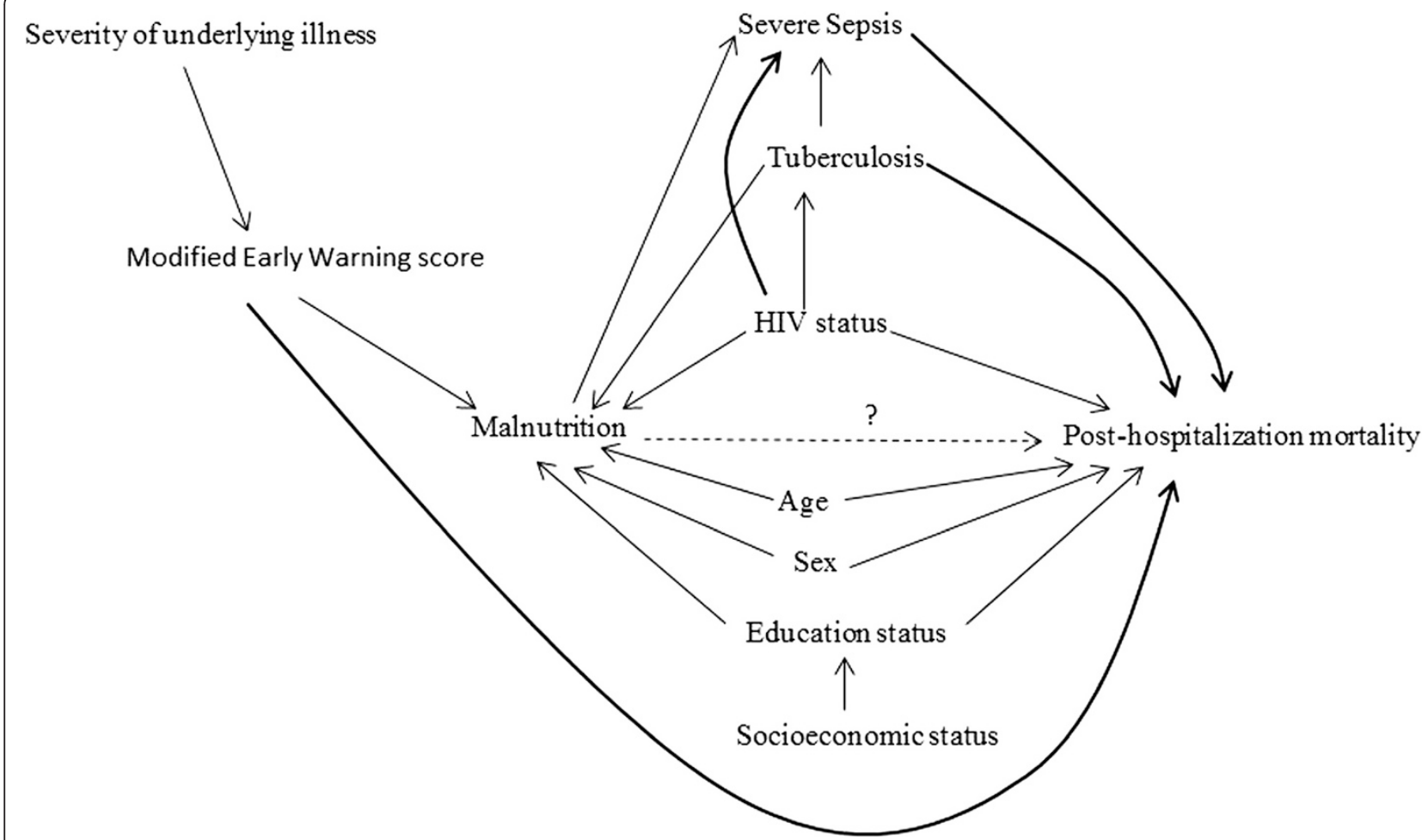

Fig. 1 A directed acyclic graph (DAG) for the causal effect of malnutrition on mortality. In this depiction, severe sepsis is a hypothesized mediator. We identified severity of underlying disease, tuberculosis, HIV infection, age, sex, and socioeconomic status as the minimum sufficient adjustment set of confounders that would require being adjusted for in order to estimate the overall effects of malnutrition on mortality. The direct effect of malnutrition on mortality is represented by the dashed line with a question mark. We hypothesized that mortality may occur through this pathway (through unknown/unmeasured mediators), or via an indirect pathway through severe sepsis. The severity of underlying illness and socioeconomic status are unmeasured constructs, which are represented by proxy measures; the modified early warning score and the education status, respectively 
sepsis, and mortality, along with the directions of hypothesized relationships. From the large conceptual framework, we selected the final adjustment variables (shown in the DAG) based on their ability to qualify as confounders of the relationships between malnutrition, severe sepsis, and mortality. Variables which in the larger framework appeared to be ancestors of malnutrition (e.g., food intake) or of severe sepsis (e.g., sepsis) but with no hypothesized independent link to mortality, as well as variables which appeared to be mediators of the association of malnutrition with mortality (e.g., anemia, with the exception of severe sepsis which was the mediator of interest), were eliminated from the final DAG (Fig. 1) and were not included in the adjusted analysis. As severity of underlying illness and socio-economic status are unmeasured, we used the MEWS as a proxy for severity of underlying illness, and education status as a proxy for socioeconomic status.

We first used a logistic regression model adjusting for the confounders to estimate the overall causal odds-ratio for the effect of malnutrition on mortality. We then used the medeff package in Stata (Stata Corp, College Station Texas) to estimate the indirect effect of malnutrition mediated through severe sepsis as well as its direct effect via other pathways $[29,30]$. In contrast to conventional logistic regression, this analysis summarizes effects as causal risk differences, with the interpretation of those differences being similar to the classical interpretation of risk comparisons on the additive scale $[10,31]$. The medeff package uses repeated simulation of unobserved counterfactual values of the mediator and outcome to obtain point estimates and confidence intervals for the mediation effect, and is thus similar to some forms of bootstrapping. As required for the validity of mediation analyses, we adjusted for confounders of the effects of both malnutrition and severe sepsis on mortality, as identified in the DAG [32]. In addition, we tested for an interaction between malnutrition and severe sepsis, which is also recommended for mediation analyses [33]. On the hypothesis that relatively mild cases of sepsis are less likely to result in death in hospital settings [1], we focused on mediation of the effects of malnutrition by severe sepsis rather than any sepsis. In assessing possible positivity violations [33], we checked overlap of each covariate as well as propensity scores for malnutrition in the malnourished and normally nourished groups.

After missing data were obtained from source documents, some patients still had missing values on suspected tuberculosis (12) fewer on GCS (12), random blood glucose (26), hemoglobin (11), white cell counts (12) and platelet counts (12). Also, 20 patients who had been discharged alive from hospital were lost to followup before 30 days. In the primary analysis, after summarizing patient characteristics by nutritional status
(Table 1), we multiply-imputed missing predictor values; we run 40 repetitions and used predictive mean matching to impute numeric variables since they had skewed distributions. We then run the analyses on the 40 post-MI datasets while averaging the results [34]. In the primary analysis, we assumed all losses to follow up to be still alive at 30 days; to assess the sensitivity of our estimate of the overall effect of malnutrition on mortality to the losses to follow-up, we repeated the analysis after further imputing the missing outcomes $[35,36]$. All analyses were performed in Stata 13.

\section{Results}

\section{Population characteristics}

We analyzed data from 318 patients. Their general characteristics have been described elsewhere [6]. Median age was 37 (interquartile range [IQR] 27 to 56), and $25 \%(n=80)$ were malnourished (by MUAC) at admission. Malnourished patients were more likely to be male (66 \% versus $47 \%, P=0.003)$, HIV positive ( $64 \%$ versus $39 \%, p<0.001)$, and more severely ill at admission (median MEWS 5, IQR 3 to 7 versus 4, IQR 2 to 6 , $P=0.005)$ compared to normally nourished patients. Malnourished patients were also more likely to have both any sepsis (66 \% versus $39 \%, p<0.001)$ and severe sepsis (51\% versus $20 \%, P<0.001)$ (Table 1 ).

\section{Unadjusted associations between selected patient characteristics and mortality}

Mortality at 30 days was $53 \%$ in those with malnutrition, and $32 \%$ in those without malnutrition. MEWS $\geq 6$ (odds ratio [OR] 2.6, $95 \%$ confidence interval [CI]:1.5, 4.6), severe sepsis (OR 2.5, 95 \% CI: 1.5, 4.1), and male sex (OR 1.8, 95 \% CI: 1.1, 2.8), predicted mortality (Table 2).

\section{Causal impact of malnutrition on mortality and mediation by severe sepsis}

After adjusting for age, sex, education status, HIV status, tuberculosis, and MEWS, patients who were malnourished at admission remained at an estimated 2.1-fold increased odds of death (Adjusted Odds-Ratio [AOR] 2.1, $95 \%$ CI: $1.2,3.7, P=0.008$ ) within 30 days of admission. This was attenuated in the sensitivity analysis using multiple imputation to account for losses to follow-up (AOR 1.8, $95 \%$ CI: 1.0, 3.1, $P=0.048$ ).

Malnourished patients were at increased odds of being severely septic (AOR 3.0, $95 \%$ CI: 1.5, 6.1, $P=0.002$ ) at admission. However, the mediation analysis showed that only a small proportion of the overall effect of malnutrition on mortality was mediated by severe sepsis (Table 3 ). Specifically, malnutrition increased the absolute risk of death by an estimated 16.8 percentage points $(95 \% \mathrm{CI}$ : $4.1,29.4$ ) overall, but only 1.1 percentage points (95\% 
Table 1 Characteristics by Nutritional Status at Admission of 318 Adults Being Hospitalized for any Medical IIIness at Mbarara Regional Referral Hospital in Uganda in April to June 2011

\begin{tabular}{|c|c|c|c|}
\hline Characteristic & Malnutrition ${ }^{a}$ present $(N=80)$ & Malnutrition absent $(N=238)$ & $P$ value \\
\hline Age, median (IQR) & $38(27 \text { to } 54)^{b}$ & 36 (27 to 56$)$ & 0.957 \\
\hline Sex male, n (\%) & $53(66)$ & $112(47)$ & 0.003 \\
\hline \multicolumn{4}{|l|}{ Education, n (\%) } \\
\hline None & $28(35)$ & $76(32)$ & 0.613 \\
\hline Primary & $43(54)$ & $116(49)$ & 0.438 \\
\hline Secondary and above & $9(11)$ & $46(19)$ & 0.098 \\
\hline HIV-infected, n (\%) & $51(64)$ & $93(39)$ & $<0.001$ \\
\hline Confirmed tuberculosis, B (\%) & $3(3.8)$ & $9(3.8)$ & 0.990 \\
\hline Sepsis, n (\%) & $53(66)$ & $93(39)$ & $<0.001$ \\
\hline Severe sepsis, n (\%) & $41(51)$ & $47(20)$ & $<0.001$ \\
\hline Temperature, median (IQR) & $37.0(36.4$ to 38.4$)$ & 37.0 (36.5 to 38.3$)$ & 0.769 \\
\hline Respiratory rate, median (IQR) & 26 (22 to 32$)$ & 25 (20 to 30$)$ & 0.277 \\
\hline Systolic blood pressure, median (IQR) & $90(85$ to 100$)$ & $110(100$ to 120$)$ & $<0.001$ \\
\hline Diastolic blood pressure, median (IQR) & 60 (50 to 67$)$ & 70 (60 to 80$)$ & $<0.001$ \\
\hline Pulse, median (IQR) & 110 (90 to 120$)$ & $100(80$ to 116$)$ & 0.006 \\
\hline Glasgow coma scale $\leq 14, \mathrm{n}(\%)$ & $15(19 \%)$ & $31(13 \%)$ & 0.282 \\
\hline \multicolumn{4}{|l|}{ Admission diagnosis, n (\%) } \\
\hline Acute infection & $24(30)$ & $73(31)$ & 0.910 \\
\hline Heart disease & $5(6.3)$ & $14(5.9)$ & 0.905 \\
\hline Kidney disease & $5(6.3)$ & $30(12.6)$ & 0.116 \\
\hline Other diagnosis & $52(65)$ & $136(57)$ & 0.216 \\
\hline MEWS, median (IQR) & 5 (3 to 7$)$ & 4 (2 to 6) & 0.003 \\
\hline Hypoglycemia, n (\%) & $12(15)$ & $25(10.5)$ & 0.342 \\
\hline Anemia, n (\%) & $35(44)$ & $68(29)$ & 0.019 \\
\hline White cell count, median (IQR) & 5.4 (3.2 to 6.2$)$ & 5.3 (3.1 to 7.2$)$ & 0.701 \\
\hline Platelet count, median (IQR) & 158 (97 to 271) & $172(117$ to 220$)$ & 0.709 \\
\hline
\end{tabular}

${ }^{a}$ Mid-upper arm circumference $<19 \mathrm{~cm}$ for females and $<20 \mathrm{~cm}$ for males

${ }^{\mathrm{b}}$ Median interquartile range unless otherwise specified

CI: -2.1, 4.4) absolute risk difference, was mediated by severe sepsis. In checking the assumptions of this analysis, we did not find evidence for interaction between malnutrition and severe sepsis $(P=0.947)$. Also, repeating the analysis while varying the number of simulations between 100 and 1000 did not lead to appreciable change in the proportion of effects mediated by severe sepsis.

\section{Discussion}

The high mortality rates among adults hospitalized for medical illness in SSA deserve concerted effort to identify points where interventions can make a difference. We used a DAG-based approach to investigate whether malnutrition affects mortality in adults hospitalized for medical illness in SSA, independent of HIV infection, tuberculosis, and severity of underlying illness, and whether this effect is mediated by severe sepsis. After adjustment for these and other confounders as identified in the DAG, we found that malnutrition increased the risk of death within 30 days of admission by 16 percentage points. This translates into needing to treat or prevent malnutrition in 7 patients in order to prevent the death of 1 patient. Although malnourished patients had increased odds of being severely septic, only $9.3 \%$ of the effect of malnutrition on mortality was mediated by severe sepsis. Our findings suggest that interventions to treat or prevent malnutrition in this population may reduce mortality and call for more studies to identify appropriate interventions.

A common definition of malnutrition has two pathophysiologic components: first, a deficiency of energy, protein, and micronutrients; and second, changes in bodily form and function [37, 38]. In general, this is where clarity ends [39-41]. Although the changes due to malnourishment are poorly understood, there is 
Table 2 Unadjusted associations between selected risk factors and mortality among 318 adults being hospitalized for any medical illness at Mbarara Regional Referral Hospital in Uganda in April to June 2011

\begin{tabular}{|c|c|c|c|}
\hline Variable & Odds ratio & $95 \% \mathrm{Cl}$ & $P$ value \\
\hline Malnutrition ${ }^{a}$ & 2.4 & $1.4,4.0$ & 0.001 \\
\hline \multicolumn{4}{|l|}{ MEWS } \\
\hline 6 to 11 & 2.6 & $1.5,4.6$ & 0.001 \\
\hline 4 to 5 & 1.4 & $0.80,2.6$ & 0.225 \\
\hline 0 to 3 & Reference & & \\
\hline Severe sepsis & 2.5 & $1.5,4.1$ & 0.001 \\
\hline Hypoglycemia ${ }^{b}$ & 1.9 & $0.97,3.6$ & 0.061 \\
\hline Confirmed TB & 1.8 & $0.55,5.6$ & 0.339 \\
\hline Male sex & 1.8 & $1.1,2.8$ & 0.017 \\
\hline Sepsis & 1.4 & $0.91,2.3$ & 0.122 \\
\hline HIV infection & 1.2 & $0.79,2.0$ & 0.348 \\
\hline Anemia $^{c}$ & 0.87 & $0.53,1.4$ & 0.594 \\
\hline \multicolumn{4}{|l|}{ Education status } \\
\hline None & 0.68 & $0.35,1.3$ & 0.265 \\
\hline Primary & 0.72 & $0.39,1.4$ & 0.306 \\
\hline Secondary or more & Reference & & \\
\hline $\mathrm{Age}^{\mathrm{d}}$ & 1.02 & $0.90,1.2$ & 0.749 \\
\hline
\end{tabular}

consensus that these changes eventually lead to bad clinical outcomes including mortality [42, 43]. Malnutrition can lead to immune suppression, possibly as a result of micronutrient or protein deficiency, hence increased susceptibility to infection $[44,45]$. Protein deficiency can also lead directly to organ dysfunction [38, 46, 47]. Reliable data on how and whether these disorders eventually lead to death, especially in adults, remain scanty, but acting via organ injury/dysfunction is plausible [48].

Potential interventions to reduce mortality from malnutrition include replacement therapy for specific deficiencies [49], treatment or prevention of infections

Table 3 Estimates of the Effect of Malnutrition on 30-day Mortality and Mediation by Severe Sepsis Among 318 Adults Being Hospitalized for any Medical IIIness at Mbarara Regional Referral Hospital in Uganda (April to June 2011)

\begin{tabular}{lcc}
\hline Parameter & Risk difference $(\%)^{\mathrm{a}}$ & $(95 \% \mathrm{Cl})$ \\
\hline Total effect & 16.8 & $4.1,29.4$ \\
Direct effect & 15.6 & $2.5,28.8$ \\
Indirect effect & 1.1 & $-2.1,4.4$ \\
\hline
\end{tabular}

Cl Confidence interval

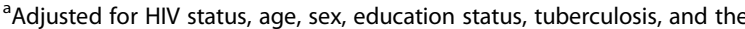
modified early warning score (MEWS) at admission
[50], treatment or removal of an underlying cause such as tuberculosis, HIV, malignancy, or chronic alcoholism [51], and correcting other disorders in the patient's physiology or anatomy that may have occurred as a result of malnutrition [52, 53]. However, the treatment of adult malnutrition remains challenging, especially in resource-limited settings. In particular, interventions aimed at reducing mortality via direct replacement of presumed deficiencies, have yielded mostly negative results [54-56]. A recent Cochrane review of up to 14 trials found that in HIV-infected adults, neither supplementary whole food, nor provision of specific supplements such as protein or micronutrients reduced mortality [54]. Possible explanations for lack of effect from such interventions may include the targeting of wrong pathways, or failure to address more immediate causes of death or important co-existing disorders. These failures may also suggest that prevention of malnutrition might be a more effective approach than its treatment.

Although we did not find evidence of substantial mediation by severe sepsis of the effect of malnutrition on mortality in the present study, our results suggest that sepsis and severe sepsis are more common in malnourished patients. Future studies should continue to investigate this association. Future studies of malnutrition-related mortality can also investigate the mediating role of other variables such as multiple organ failure, hypoglycemia, and hypothermia, which may result from malnutrition [15] and have been associated with mortality [57]. Studies can also assess whether damage to tissues from malnutrition [48] may require independent interventions. Such studies have potential to identify supportive treatments that might yield survival benefit to patients with malnutrition.

Our study has some limitations. We were unable to establish 30-day vital status for $7.8 \%$ of normally nourished patients and $2.5 \%$ of malnourished patients. However, our sensitivity analysis using multiple imputations of the missing outcomes did not qualitatively alter our overall conclusions. Measurement of malnutrition is also complicated by absence of a gold standard and imperfect diagnostics. However, as MUAC has high specificity, its low sensitivity was expected to introduce minimal bias, given that measurement bias in a cohort study mostly depends on specificity [58]. Residual confounding of the effects of both malnutrition and severe sepsis may also arise from imperfect measurement of severity of underlying illness and tuberculosis. Also, diagnoses of infections, as well as focus of infection, were based on clinical suspicion and physical examination as confirmation of such diagnoses by laboratory or radiological means was not always possible due to resourcelimitations in this setting. However, all clinical diagnoses were confirmed by more than one clinician, which may 
increase their accuracy. Finally, we were unable to assess the association between malnutrition and mortality in further detail among patients with HIV infection due to sample size limitations. In particular, it would be important to assess whether among HIV-infected patients malnutrition remains associated with mortality even after adjusting for level of immunosuppression or whether this association varies by level of immunosuppression (for example, as measured by the CD4+ T-cell count). Future studies focusing on these questions are recommended.

\section{Conclusions}

In conclusion, malnutrition increased mortality in predominantly young and middle aged adults hospitalized for medical illness in sub-Saharan Africa. Interventions to treat or prevent malnutrition in this population may therefore reduce mortality. Although severe sepsis only very weakly mediated the effect of malnutrition on mortality, our results add substantially to the hypothesis that malnourished patients are more likely to have severe sepsis. We recommend future studies to further explore these pathways in order to guide effective interventions against mortality.

\section{Abbreviations}

AOR: Adjusted odds ratio; Cl: Confidence interval; DAG: Directed acyclic graph; GCS: Glasgow coma score; IQR: Interquartile range; MEWS: Modified early warning score; MUAC: Mid-upper arm circumference; SSA: Sub-Saharan Africa; TB: Tuberculosis.

\section{Competing interests}

The authors have no conflicts of interest to declare.

\section{Authors' contributions}

SBA, conceptualized the study, prepared and analyzed the data, wrote and edited the manuscript. AA conceptualized the study, wrote and edited the manuscript. EV conceptualized the study, prepared and analyzed the data, and wrote and edited the manuscript. EV also provided mentorship to SBA on statistical methods related to this analysis. CM conceptualized the study, and wrote and edited the manuscript. All authors provided important feedback on the manuscript and approved its final version.

\section{Acknowledgement}

The primary study via which these data were collected was conducted with support from the University of Virginia, Charlottesville, Center for Global Health - Pfizer Initiative in International Health.

\section{Author details}

'Department of Medicine, Mbarara Regional Referral Hospital, P.O Box, 40, Mbarara, Uganda. ${ }^{2}$ Department of Medicine, Mbarara University of Science and Technology, Mbarara, Uganda. ${ }^{3}$ Department of Epidemiology and Biostatistics, University of California, San Francisco, CA, USA.

Received: 10 November 2014 Accepted: 8 October 2015 Published online: 10 December 2015

\section{References}

1. Ssekitoleko R, Pinkerton R, Muhindo R, Bhagani S, Moore CC. Aggregate evaluable organ dysfunction predicts in-hospital mortality from sepsis in Uganda. Am J Trop Med Hyg. 2011;85(4):697-702.

2. Asiimwe SB, Okello S, Moore CC. Frequency of vital signs monitoring and its association with mortality among adults with severe sepsis admitted to a general medical ward in Uganda. PLoS One. 2014;9(2):e89879.
3. Andrews B, Muchemwa L, Kelly P, Lakhi S, Heimburger DC, Bernard GR. Simplified severe sepsis protocol: a randomized controlled trial of modified early goal-directed therapy in Zambia. Crit Care Med. 2014;42(11):2315-24.

4. Niyongabo T, Henzel D, Ndayishimyie JM, Melchior JC, Ndayiragije A, Ndihokubwayo JB, et al. Nutritional status of adult inpatients in Bujumbura, Burundi (impact of HIV infection). Eur J Clin Nutr. 1999;53(7):579-82.

5. Jacob ST, Moore CC, Banura P, Pinkerton R, Meya D, Opendi P, et al. Severe sepsis in two Ugandan hospitals: a prospective observational study of management and outcomes in a predominantly HIV-1 infected population. PLoS One. 2009;4(11):e7782.

6. Asiimwe SB, Muzoora C, Wilson LA, Moore CC. Bedside measures of malnutrition and association with mortality in hospitalized adults. Clin Nutr. 2015;34(2):252-6.

7. Melchior JC, Niyongabo T, Henzel D, Durack-Bown I, Henri SC, Boulier A. Malnutrition and wasting, immunodepression, and chronic inflammation as independent predictors of survival in HIV-infected patients. Nutrition. 1999;15(11-12):865-9.

8. Shrier I, Platt RW. Reducing bias through directed acyclic graphs. BMC Med Res Methodol. 2008:8:70.

9. Nyamogoba HD, Mbuthia G, Mining S, Kikuvi G, Biegon R, Mpoke S, et al. HIV co-infection with tuberculous and non-tuberculous mycobacteria in western Kenya: challenges in the diagnosis and management. Afr Health Sci. 2012;12(3):305-11.

10. VanderWeele TJ. Causal mediation analysis with survival data. Epidemiology. 2011;22(4):582-5.

11. Felblinger DM. Malnutrition, infection, and sepsis in acute and chronic illness. Crit Care Nurs Clin North Am. 2003;15(1):71-8.

12. Katona P, Katona-Apte J. The interaction between nutrition and infection. Clin Infect Dis. 2008:46(10):1582-8.

13. Kahigwa E, Schellenberg D, Sanz S, Aponte JJ, Wigayi J, Mshinda H, et al. Risk factors for presentation to hospital with severe anaemia in Tanzanian children: a case-control study. Trop Med Int Health. 2002;7(10):823-30.

14. Schneider SM, Veyres P, Pivot X, Soummer AM, Jambou P, Filippi J, et al. Malnutrition is an independent factor associated with nosocomial infections. Br J Nutr. 2004;92(1):105-11.

15. Kerpel-Fronius E. The main causes of death in malnutrition. Acta Paediatr Hung. 1984;25(1-2):127-30.

16. Roy SK, Buis M, Weersma R, Khatun W, Chowdhury S, Begum A, et al. Risk factors of mortality in severely-malnourished children hospitalized with diarrhoea. J Health Popul Nutr. 2011;29(3):229-35.

17. Fondu P, Hariga-Muller C, Mozes N, Neve J, Van Steirteghem A, Mandelbaum IM. Protein-energy malnutrition and anemia in Kivu. Am J Clin Nutr. 1978;31(1):46-56.

18. Hicks RM, Padayatchi N, Shah NS, Wolf A, Werner L, Sunkari VB, et al. Malnutrition associated with unfavorable outcome and death among South African MDR-TB and HIV co-infected children. Int J Tuberc Lung Dis. 2014;18(9):1074-83.

19. Ferro-Luzzi A, James WP. Adult malnutrition: simple assessment techniques for use in emergencies. Br J Nutr. 1996;75(1):3-10.

20. James WP, Mascie-Taylor GC, Norgan NG, Bistrian BR, Shetty PS, Ferro-Luzzi A. The value of arm circumference measurements in assessing chronic energy deficiency in Third World adults. Eur J Clin Nutr. 1994;48(12):883-94.

21. Powell-Tuck J, Hennessy EM. A comparison of mid upper arm circumference, body mass index and weight loss as indices of undernutrition in acutely hospitalized patients. Clin Nutr. 2003;22(3):307-12

22. Jeyakumar A, Ghugre P, Swapnil G. Mid-Upper-Arm Circumference (MUAC) as a Simple measure to assess the nutritional status of adolescent girls as compared with BMI. ICAN. 2013;5:22.

23. Dellinger RP, Levy MM, Rhodes A, Annane D, Gerlach H, Opal SM, et al. Surviving Sepsis Campaign: international guidelines for management of severe sepsis and septic shock, 2012. Intensive Care Med. 2013;39(2):165-228.

24. Bagshaw SM, Bellomo R, Jacka MJ, Egi M, Hart GK, George C, et al. The impact of early hypoglycemia and blood glucose variability on outcome in critical illness. Crit Care. 2009;13(3):R91.

25. The World Health Organization. Haemoglobin concentrations for the diagnosis of anaemia and assessment of severity. In: Vitamin and Mineral Nutrition Information System. Geneva: World Health Organization; 2011.

26. Koole O, Munthali L, Mhango B, Mpunga J, Glynn JR, Crampin AC. Impact of changing diagnostic criteria for smear-positive tuberculosis: a cohort study in Malawi. Int J Tuberc Lung Dis. 2014;18(7):843-6. 
27. Subbe CP, Kruger M, Rutherford P, Gemmel L. Validation of a modified Early Warning Score in medical admissions. QJM. 2001;94(10):521-6.

28. Kyriacos $U$, Jelsma J, James M, Jordan S. Monitoring vital signs: development of a modified early warning scoring (MEWS) system for general wards in a developing country. PLoS One. 2014;9(1):e87073.

29. Hicks R, Tingley D. Causal mediation analysis. Stata J. 2011;11(4):605-19.

30. Imai K, Keele L, Tingley D. A general approach to causal mediation analysis. Psychol Methods. 2010;15(4):309-34

31. Grotta A, Bellocco R. A review of mediation analysis in Stata: principles, methods and applications. Firenze: Italian Stata Users Group Meeting; 2013.

32. Cole SR, Hernan MA. Fallibility in estimating direct effects. Int J Epidemiol. 2002;31(1):163-5.

33. Pearl J. The causal mediation formula-a guide to the assessment of pathways and mechanisms. Prev Sci. 2012;13(4):426-36.

34. Morris TP, White IR, Royston P. Tuning multiple imputation by predictive mean matching and local residual draws. BMC Med Res Methodol. 2014;14:75.

35. Vonta F, Karagrigoriou A. Variable selection strategies in survival models with multiple imputations. Lifetime Data Anal. 2007;13(3):295-315.

36. Nunes LN, Kluck MM, Fachel JM. [Multiple imputations for missing data: a simulation with epidemiological data]. Cad Saude Publica. 2009;25(2):268-78.

37. Kinosian B, Jeejeebhoy KN. What is malnutrition? Does it matter? Nutrition. 1995;11(2 Suppl):196-7.

38. Waller EG, Wade AJ, Treasure J, Ward A, Leonard T, Powell-Tuck J. Physical measures of recovery from anorexia nervosa during hospitalised re-feeding. Eur J Clin Nutr. 1996:50(3):165-70.

39. Gopalan S. Malnutrition: causes, consequences, and solutions. Nutrition. 2000;16(7-8):556-8.

40. Casey KM. Malnutrition associated with HIV/AIDS. Part One: Definition and scope, epidemiology, and pathophysiology. J Assoc Nurses AIDS Care. 1997;8(3):24-32

41. Blössner $M$, de Onis M. Malnutrition: Quantifying the health impact at national and local levels. Geneva: The World Health Organization; 2005.

42. Saunders J, Smith T. Malnutrition: causes and consequences. Clin Med. 2010;10(6):624-7.

43. Muller $\mathrm{O}$, Krawinkel M. Malnutrition and health in developing countries. CMAJ. 2005;173(3):279-86.

44. Perronne C. Tuberculosis, HIV infection, and malnutrition: an infernal trio in central Africa. Nutrition. 1999;15(4):321-2.

45. Santos JI, Arredondo JL, Vitale JJ. Nutrition, infection and immunity. Pediatr Ann. 1983;12(3):182-94.

46. Shaw AD, Vail GM, Haney DJ, Xie J, Williams MD. Severe protein C deficiency is associated with organ dysfunction in patients with severe sepsis. J Crit Care. 2011;26(6):539-45.

47. Cederholm T, Jagren C, Hellstrom K. Nutritional status and performance capacity in internal medical patients. Clin Nutr. 1993;12(1):8-14.

48. Eisenhut M. Malnutrition causes a reduction in alveolar epithelial sodium and chloride transport which predisposes to death from lung injury. Med Hypotheses. 2007;68(2):361-3.

49. McMahon MM, Bistrian BR. The physiology of nutritional assessment and therapy in protein-calorie malnutrition. Dis Mon. 1990;36(7):373-417.

50. Suttmann U, Muller MJ, Ockenga J, Hoogestraat L, Coldewey R, Schedel I, et al. Malnutrition and immune dysfunction in patients infected with human immunodeficiency virus. Klin Wochenschr. 1991;69(4):156-62.

51. Marais BJ, Lonnroth K, Lawn SD, Migliori GB, Mwaba P, Glaziou P, et al. Tuberculosis comorbidity with communicable and non-communicable diseases: integrating health services and control efforts. Lancet Infect Dis. 2013;13(5):436-48.

52. Drake WM, Winter TA, Price SK, O'Keefe SJ. Small bowel intussusception and brown bowel syndrome in association with severe malnutrition. Am J Gastroenterol. 1996;91(7):1450-2.

53. Shepherd A. Nutrition support 1: risk factors, causes and physiology of malnutrition. Nurs Times. 2009;105(4):18-20.

54. Grobler L, Siegfried N, Visser ME, Mahlungulu SS, Volmink J. Nutritional interventions for reducing morbidity and mortality in people with HIV. Cochrane Database Syst Rev. 2013;2:CD004536.

55. van den Broek NR, White SA, Flowers C, Cook JD, Letsky EA, Tanumihardjo $\mathrm{SA}$, et al. Randomised trial of vitamin A supplementation in pregnant women in rural Malawi found to be anaemic on screening by HemoCue. BJOG. 2006;113(5):569-76.
56. Dennis MS, Lewis SC, Warlow C, Collaboration FT. Routine oral nutritional supplementation for stroke patients in hospital (FOOD): a multicentre randomised controlled trial. Lancet. 2005;365(9461):755-63.

57. Ssekitoleko R, Jacob ST, Banura P, Pinkerton R, Meya DB, Reynolds SJ, et al. Hypoglycemia at admission is associated with inhospital mortality in Ugandan patients with severe sepsis. Crit Care Med. 2011;39(10):2271-6.

58. Copeland KT, Checkoway H, McMichael AJ, Holbrook RH. Bias due to misclassification in the estimation of relative risk. Am J Epidemiol. 1977;105(5):488-95.

\section{Submit your next manuscript to BioMed Central and take full advantage of:}

- Convenient online submission

- Thorough peer review

- No space constraints or color figure charges

- Immediate publication on acceptance

- Inclusion in PubMed, CAS, Scopus and Google Scholar

- Research which is freely available for redistribution 